\title{
Ultra Wide X-Band Microwave Imaging of Concealed Weapons and Explosives Using 3D-SAR Technique
}

\author{
P. Millot and L. Casadebaig \\ ONERA, The French Aerospace Lab, Electromagnetism and Radar Department (DEMR), \\ BP74025, 2 avenue E. Belin, 31055 Toulouse, France \\ Correspondence should be addressed to P. Millot; patrick.millot@onera.fr
}

Received 19 December 2014; Revised 2 April 2015; Accepted 14 April 2015

Academic Editor: Lorenzo Crocco

Copyright (C) 2015 P. Millot and L. Casadebaig. This is an open access article distributed under the Creative Commons Attribution License, which permits unrestricted use, distribution, and reproduction in any medium, provided the original work is properly cited.

In order to detect and image concealed weapons and explosives, an electromagnetic imaging tool with its related signal processing is presented. The aim is to penetrate clothes and to find personal-born weapons and explosives under clothes. The chosen UWB frequency range covers the whole X-band. The frequency range is justified after transmission measurements of numerous clothes that are dry or slightly wet. The apparatus and the 3D near-field SAR processor are described. A strategy for contour identification is presented with results of some simulants of weapon and explosive. A conclusion is drawn on the possible future of this technique.

\section{Introduction}

The detection of concealed weapons or concealed explosives has now become a major issue. There is a growing need to keep secure areas in airports, subways, and so forth. Weapons and explosives can be carried by people in luggage and also hidden under their clothes.

Many detection techniques have been proposed for the detection of the presence of concealed weapons. Conventional techniques are X-rays and metal detectors. Nevertheless, X-rays can be dangerous for human health. Metal detectors are not imager sensors and anyway cannot detect nonmetallic objects. Terahertz waves are now also emerging techniques for vision through matter, as well as millimeter wave imagers [1,2].

We propose in this paper to operate in X-band (8.212.4 GHz) and to use an Ultra Wide Band (UWB) frequency sweep for electromagnetic imaging. It is well known that, for an electromagnetic imaging sensor, the image resolution increases with frequency. This is why the use of the highest frequencies seems the most interesting. But this argument considered alone is a bit simplistic, and there is a compromise to be found between resolution and penetration in matter.
The expected benefits of using microwaves instead of millimeter waves are the following:

(i) Wave attenuation in "through-the-cloth" propagation conditions increases with frequency. So we expect very low electromagnetic losses when crossing clothes (even when they are very thick or sweaty).

(ii) UWB microwaves are well suited for imaging and deliver a highly resolved image. On the contrary, there is no risk of entering into the person intimacy because images do not look like optical images.

(iii) There may be less "clutter" or "speckle" due to roughness (of clothes, of objects) at microwave frequencies.

(iv) One can imagine building in a near future an inexpensive tool due to low cost microwave components in X-band. One can use also standard antennas like horn antennas.

\section{Experimental Study of Transmission through Clothing}

Transmission measurements of microwaves through clothes have been performed in order to assess the frequency 


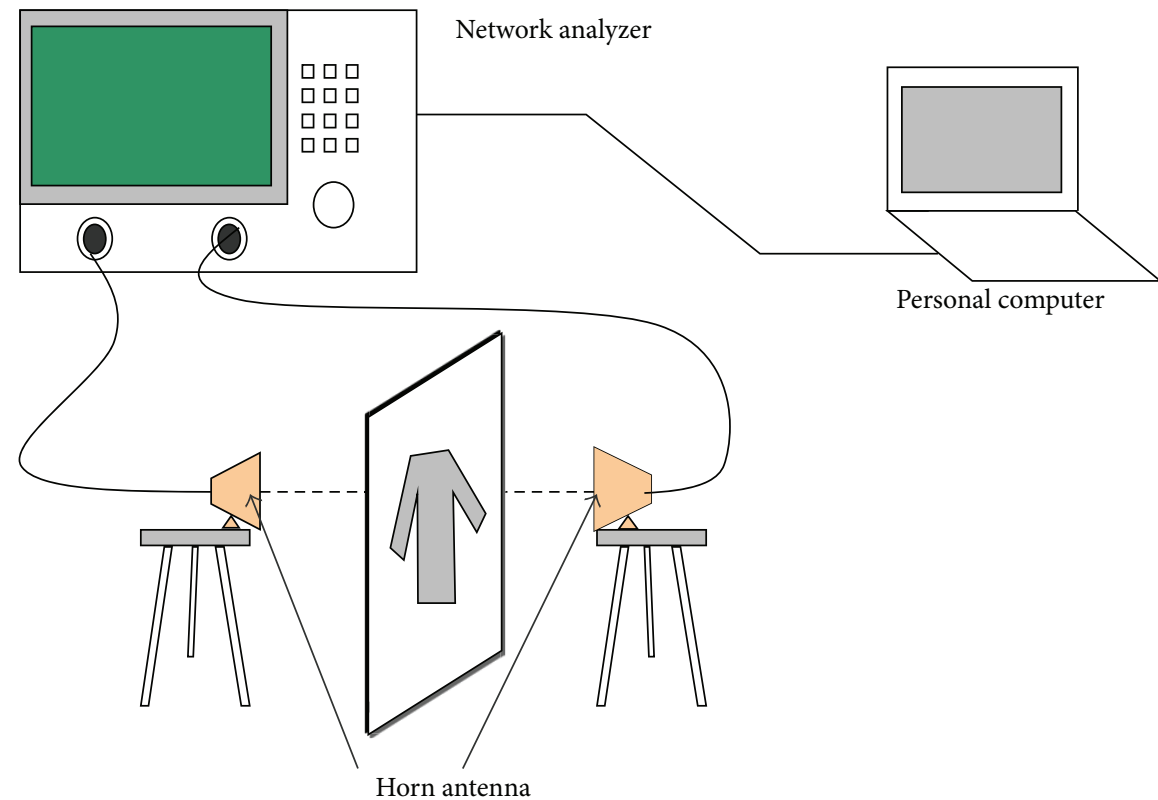

FIGURE 1: Microwave test bench for transmission measurements through clothing.

choice. To perform these measurements, a network analyzer (Figure 1) is used. The transmission has been measured between two horn antennas (using the parameter $S_{12}$ ). For each garment, frequency measurements have been made by sweeping in frequency from 0.3 to $40 \mathrm{GHz}$.

Results are summarized in Table 1 and are given with respect to two criteria:

(i) Maximum frequency for which the attenuation is negligible (less than $1 \mathrm{~dB}$ for one path).

(ii) Maximum attenuation in the $1-40 \mathrm{GHz}$ bandwidth.

(Note that these results give only some magnitudes and that attenuation versus frequency curves would show only measurement errors.)

As one can notice, dry clothes do not cause a high attenuation for frequencies up to $40 \mathrm{GHz}$. The frequency range for an attenuation that can be totally neglected is roughly $(1-16 \mathrm{GHz})$. The effect of the presence of human sweat on underwear has also been considered of experimental way. In order to quantify it, a cotton T-shirt sprayed with salted water has been put under test, using the same percentage as the human sweat, that is to say, $3.5 \%$ salt. The results are given in Figure 2.

In conclusion, underwear "wet by human sweat" can present typically $5 \mathrm{~dB}$ of attenuation at frequencies exceeding $10 \mathrm{GHz}$ and so $10 \mathrm{~dB}$ for the round trip. This value may remain acceptable, depending on the sensitivity of the final detector.

\section{UWB Microwave Imaging Tool Hardware}

In order to estimate the shape of the desired object, it is necessary to perform three-dimensional microwave imaging. As one knows, three-dimensional imaging is the best solution
TABLE 1: Results on transmission tests on clothes.

\begin{tabular}{|c|c|c|}
\hline Garments & $\begin{array}{l}\text { Max. frequency } \\
\text { for which } \\
\text { attenuation } \\
<1 \mathrm{~dB}\end{array}$ & $\begin{array}{c}\text { Max. } \\
\text { attenuation in } \\
\text { the whole } \\
1-40 \mathrm{GHz} \text { band }\end{array}$ \\
\hline $\begin{array}{l}\# 1 \text { leather jacket }(100 \% \\
\text { leather) }\end{array}$ & $20 \mathrm{GHz}$ & $1 \mathrm{~dB}$ \\
\hline $\begin{array}{l}\# 2 \text { cotton shirt }(100 \% \\
\text { cotton) }\end{array}$ & $16 \mathrm{GHz}$ & $2 \mathrm{~dB}$ \\
\hline $\begin{array}{l}\# 3 \text { thick sweater ( } 50 \% \text { wool, } \\
50 \% \text { acrylic) }\end{array}$ & $20 \mathrm{GHz}$ & $1 \mathrm{~dB}$ \\
\hline $\begin{array}{l}\text { \#4 lightweight wool jacket } \\
\text { (100\% wool) }\end{array}$ & $20 \mathrm{GHz}$ & $1 \mathrm{~dB}$ \\
\hline $\begin{array}{l}\# 5 \text { shirt }(80 \% \text { cotton and } \\
20 \% \text { polyester })\end{array}$ & $20 \mathrm{GHz}$ & $1 \mathrm{~dB}$ \\
\hline $\begin{array}{l}\text { \#6 parka (waterproof } \\
\text { canvas filled) }\end{array}$ & $17 \mathrm{GHz}$ & $2 \mathrm{~dB}$ \\
\hline \#7 wool jacket (100\% wool) & $16.5 \mathrm{GHz}$ & $1 \mathrm{~dB}$ \\
\hline $\begin{array}{l}\# 8 \text { waterproof jacket (inner } \\
\text { side: } 100 \% \text { cotton; outer } \\
\text { side: } 100 \% \text { polyester) }\end{array}$ & $16 \mathrm{GHz}$ & $2 \mathrm{~dB}$ \\
\hline
\end{tabular}

in terms of object identification because the sizes (in $X, Y$ ) and "depth" (in $Z$, parallel to the bore sight) of the object can be retrieved (see Figure 3).

A bidimensional synthetic antenna is required for the imaging process. The best solution for real-time measurements would be to build an UWB bidimensional array of antennas (see Figure 4, on the left). When the number of antennas is large, real-time operations can be obtained by antenna switching within an antenna array. 


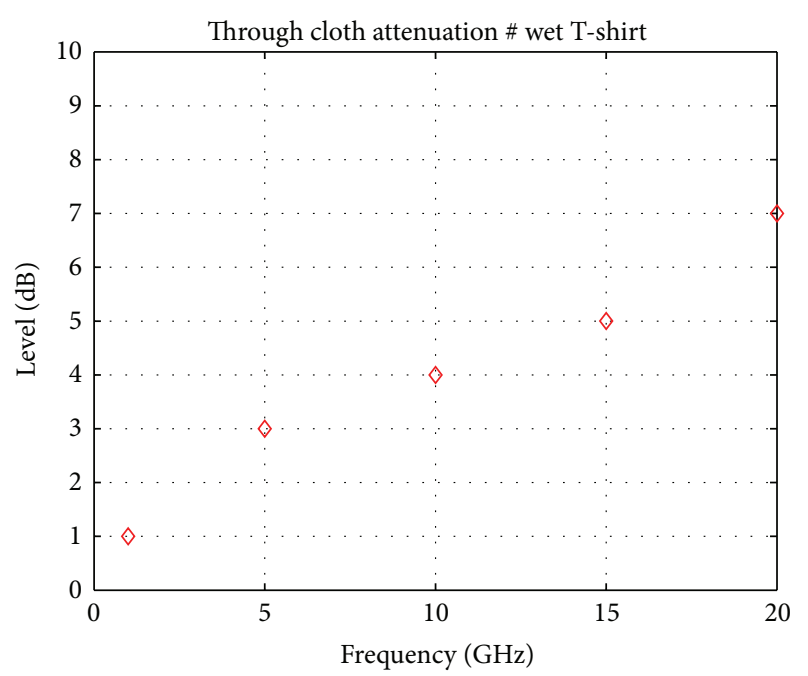

Figure 2: Attenuation versus frequency for a wet T-shirt.

However, in the framework of this research, the solution of the displacement of a single antenna that simulates a synthetic aperture has been adopted. This simple technique of scanning a plane surface simulates an array of antennas. In fact, only one antenna is needed and the microwave device is quite simple (see Figure 4, on the right).

\section{Near-Field Imaging Using 3D-SAR Algorithm}

Inverse scattering considering the $3 \mathrm{D}$ nature is a promising field for dielectric objet identification [3]. For this purpose, many algorithms in the near-field configuration have been developed [4, 5]. For microwave imaging, we have used SAR (synthetic aperture radar) imaging. Because we have achieved the experimental conditions of the antenna synthesis, UWB SAR imaging has become very performing. The idea is to adopt algorithms that are fast and robust to noise and to artifacts coming from the standing waves.

SAR imaging is based upon back-propagation principle and is analogous to Kirchhoff's migration. Due to the length of the synthetic antenna and the distance to the object $(40 \mathrm{~cm}$ for both), we are in near field of the synthetic antenna and so special algorithms derived from spot-light SAR or seismic migration can be used. Basically, the radar image is built by summing delayed signals acquired for a very large number of antennas and frequencies. The frequency formulation is based on phase shifts compensations [6]. The 2D algorithm is also explained in [7] and an extension to 3D is now proposed.

Let $S_{r}$ be the complex signal recorded for antenna points $\left(x_{i}, y_{l}\right)$ (number of $\left.N_{x} N_{y}\right)$ for a set of $N_{f}$ frequencies. Then the local reflectivity $\gamma$ of the target at the point $M(x, y, z)$ can be computed as

$$
\gamma(x, y, z)=\left|\alpha \sum_{i=1}^{N_{x}} \sum_{l=1}^{N_{y}} \sum_{k=1}^{N_{F}} W\left(x_{i}, y_{l}, x, y, f_{k}\right) S_{r}^{*}\left(x_{i}, y_{l,} f_{k}\right) d^{2}\left(x-x_{i}, y-y_{k}, z\right) e^{-j\left(4 \pi f_{k} / c\right) d\left(x-x_{i}, y-y_{k}, z\right)}\right|,
$$

where $\alpha$ is a proportionality factor depending on normalization, $x_{i}, y_{l}$ are the coordinates of the antenna points (total number $N_{x} * N_{y}$ ), $f_{k}$ is the current frequency (total number $\left.N_{F}\right), d$ is the distance from antenna to the current point $(x, y)$, and $S_{r}^{*}$ denotes the complex conjugate of $S_{r}$.

$W$ is supposed to be a $3 \mathrm{D}$ weight function to achieve apodization and antenna gain deconvolution:

$$
W\left(x_{i}, y_{l}, x, y, f_{k}\right)=\frac{\operatorname{Ham} 3\left(f, x_{i}, y_{l}\right)}{f_{k} G\left(x_{i}, y_{l}, x, y, f_{k}\right)} .
$$

Ham 3 designs the 3D Hamming functions in frequency and space used to reduce side-lobes in the 3 dimensions (2) and $G$ is the antenna gain factor. The chosen antenna is a pyramidal horn of $15 \mathrm{~dB}$ gain and of 30 -degree $3 \mathrm{~dB}$ beamwidth. In practice, it has been found that the weight function along $x$ and $y$ is unnecessary as the antenna pattern furnishes a kind of natural apodization and the frequency scaling is a smooth function. The $d^{2}$ multiplication in (1) is also quite ineffective as the distance does not vary rapidly on the contrary of the phase term.

The space sampling requirement is at least $\lambda / 4$ for SAR processing. Frequency sampling must also respect the criterion of antialiasing in range domain. This leads to a hundred of frequency samples. Typical figures are then 100 antenna positions and 100 frequency steps. This is why the basic algorithm of (1) using three sums has a computation time of around 10 minutes for a cube of $0.5 \times 0.5 \times 0.5=0.125 \mathrm{~m}^{3}$. In order to improve the computation efficiency, we have developed a new algorithm with another formulation. We have thought to use the $2 \mathrm{D}$ Fast Fourier Transform $\left(\mathrm{FFT}_{2}\right)$ algorithm for computation [8]. Let us construct $M$ as the matrix of measured data of dimensions $N_{x} * N_{y} * N_{f}$. In fact, squared matrices of dimensions $N_{a}^{2}$ (number of antenna data points) are stacked along the frequency dimension in order to create the large matrix $M$. Then, a "focusing operator" Foc is computed, knowing the geometry of the radar scene $(x, y, z)$ and the synthetic aperture $\left(x_{a}, y_{a}\right)$. It is computed once and then stored for all. The existence of such an operator is due to the formalism of the double convolution in $x$ and $y$ for the focusing processing. This means that the local reflectivity $\gamma(x, y, z)$ instead of (1) can be expressed as

$$
\begin{aligned}
& \gamma(x, y, z)=\sum_{f}\left[M\left(x, x_{a}, y, y_{a}, z, f\right)\right. \\
& \left.* * \operatorname{Foc}\left(x, x_{a}, y, y_{a}, z, f\right)\right] \\
& =\sum_{f}\left[\operatorname { F F T } _ { 2 } ^ { - 1 } \left[\operatorname{FFT}_{2}\left[M\left(x, x_{a}, y, y_{a}, z, f\right)\right]\right.\right. \\
& \left.\left.\cdot \operatorname{FFT}_{2}\left[\operatorname{Foc}\left(x, x_{a}, y, y_{a}, z, f\right)\right]\right]\right] .
\end{aligned}
$$




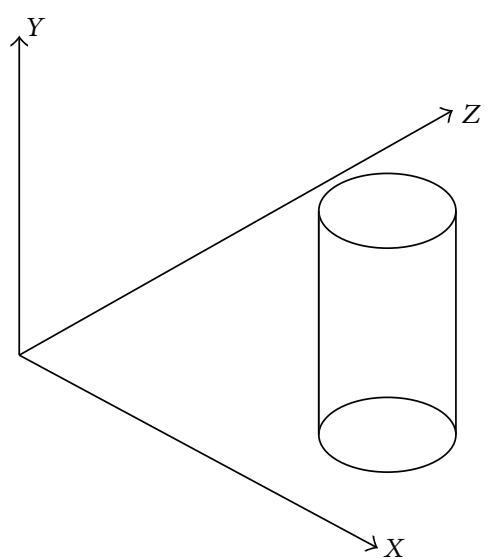

FIGURE 3: Target geometry.

Using the 2D-FFT (Two-Dimensional Fast Fourier Transform) algorithm for the Fourier Transform in two dimensions over the $(x, y)$ coordinates, the imaging algorithm (see (3)) became very efficient. The 3D-SAR computation time for a pixel of $1 \mathrm{~cm}$ and a volume of $0.125 \mathrm{~m}^{3}$ with $N_{a}=51$ and $N_{f}=201$ was about 2 seconds in MATLAB 8 on a standard PC.

\section{Experimental Results}

The "radar tool" is a versatile microwave imaging device with very high spatial resolution, due to the combination of Ultra Wide Bandwidth and large synthetic aperture. The microwave electronics is based on a portable Network Vector Analyzer (NVA) used both as transmitter and as receiver. It is linked to a PC equipped with the MATLAB 8 software. The chosen configuration for the measurement is the $S_{11}$ /reflection parameter in frequency domain. Thus, only one antenna is used for transmitting and receiving. This is to avoid bistatic angular shifts in case of two separated antennas operating in near field. The bidimensional synthetic aperture $(X, Y)$ is synthetized with the help of a two-axis translator (Figure 5). The transmitted power is $1 \mathrm{~mW}$. The space sampling is $7.5 \mathrm{~mm}$ which corresponds to a quarter of the mean carrier wavelength $(3 \mathrm{~cm}$ at $10 \mathrm{GHz})$. The overall dimensions for the synthetic aperture in both directions are $375 \mathrm{~mm}$ and $375 \mathrm{~mm}$. This value has been carefully selected as a compromise between image resolution and measurement time. This means that the real image resolution is roughly $3 \mathrm{~cm}$ in $X$ and $Y$ direction at $40 \mathrm{~cm}$ of distance between the antenna and the target. Along $Z$, the resolution is fixed by the frequency bandwidth and is about $4 \mathrm{~cm}$.

Table 2 lists the specifications of "MIXIM", the microwave imager.

The three chosen objects for the test measurement are described in Table 3.

The measurements are made with a different object at each time. The objects are suspended between the coat (item \#6) and an electromagnetic flat absorber that simulates the human body. As it is not possible to take a human being
TABLE 2: Specifications of the microwave imager.

\begin{tabular}{lc}
\hline \multicolumn{2}{c}{ MIXIM (microwave X-band imager) } \\
\hline Operating frequency & $8.2-12.4 \mathrm{GHz}$ \\
Number of frequency samples & 201 \\
Transmitted power & $1 \mathrm{~mW}$ \\
$T_{x} / R_{x}$ antenna & Pyramidal horn with $15 \mathrm{~dB}$ gain \\
Video bandwidth & $30 \mathrm{KHz}$ \\
Synthetic aperture length & $375 \mathrm{~mm}$ \\
Number of antenna samples & 2601 \\
$x$-axis step & $7.5 \mathrm{~mm}$ \\
$y$-axis step & $7.5 \mathrm{~mm}$ \\
Total measurement time & $24 \mathrm{minutes}$ \\
$X, Y$ resolution & $3 \mathrm{~cm}$ \\
$Z$ resolution & $4 \mathrm{~cm}$ \\
\hline
\end{tabular}

because of the measurement time, we simulate like this the wave absorption by the human being and the reflection on its surface could be similar to that of human fat. The distance between the antenna and the object is $40 \mathrm{~cm}$ in order to keep the nominal resolution of around $3 \mathrm{~cm}$ (see Figure 6).

The imaging results are now presented. First, we have obtained 3D images using the signal processing algorithm. 3D images can be seen as a set of 2D images (or cuts), for instance, taken in the vertical plane. One can choose a $2 \mathrm{D}$ image in the vertical plane at random. But we have adopted the following better strategy: in order to detect the object, we remove the mean value of each $2 \mathrm{D}$ image and we keep the pixels that are above this value. Then we take 10 vertical cuts separated by $1 \mathrm{~cm}$ around the range of $40 \mathrm{~cm}$. These $2 \mathrm{D}$ images are summed in intensity, normalized to the maximum value, and displayed with a color code in intensity between $30 \%$ and $100 \%$ of the strongest pixel.

The first image (Figure 7) represents the ceramic knife under cloth \#6. The handle is metal (on the left), and the blade is ceramic (on the right). The strongest local reflectivity is on the metal (note that the handle is cambered) but the dielectric blade is clearly visible because it is flat. The dielectric constant of ceramic is estimated to 9 .

The second object is a plastic toy gun under the same cloth. From Figure 8, one can clearly imagine the shape of this object.

The third object is the sand pouch in vertical position. We have selected very fine sand coming from a beach in order to simulate an explosive mass. Its dielectric constant is 2.6. From Figure 9, one can estimate the cross section of explosive mass. When the explosive is thick enough (say $10 \mathrm{~cm}$ ), we can estimate its thickness and so the volume. Of course, explosive appears here only as a large "anomaly," which is enough to trigger an alert.

For objects of $4 \mathrm{~cm}$ size, the imager will deliver just a spot which can be satisfactory for detection but not for shape identification. This is why we claim that the dimensions should be at least $10 \mathrm{~cm}$ for better shape estimation.

An extra measurement has been made for the case of the ceramic knife under a wet shirt (slightly watered with a spray of salted water in order to simulate human sweat). 


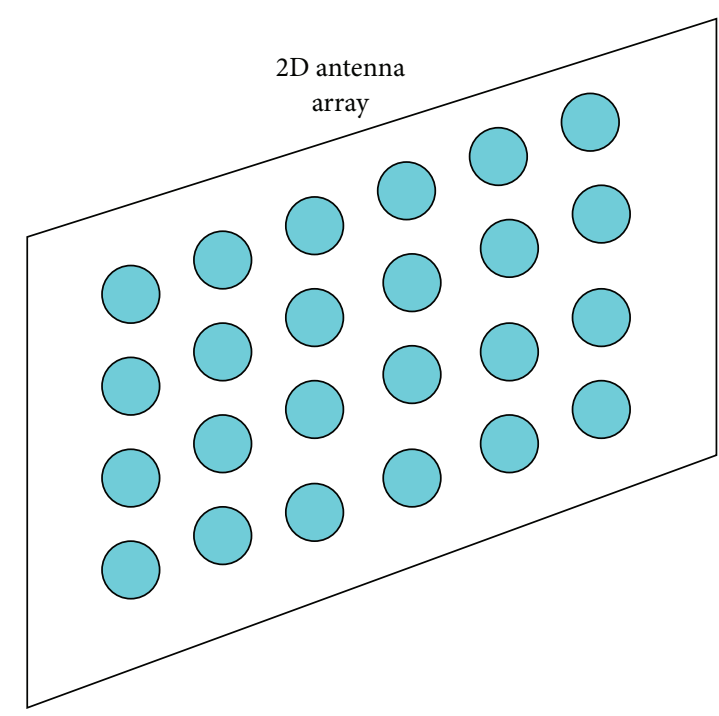

(a)

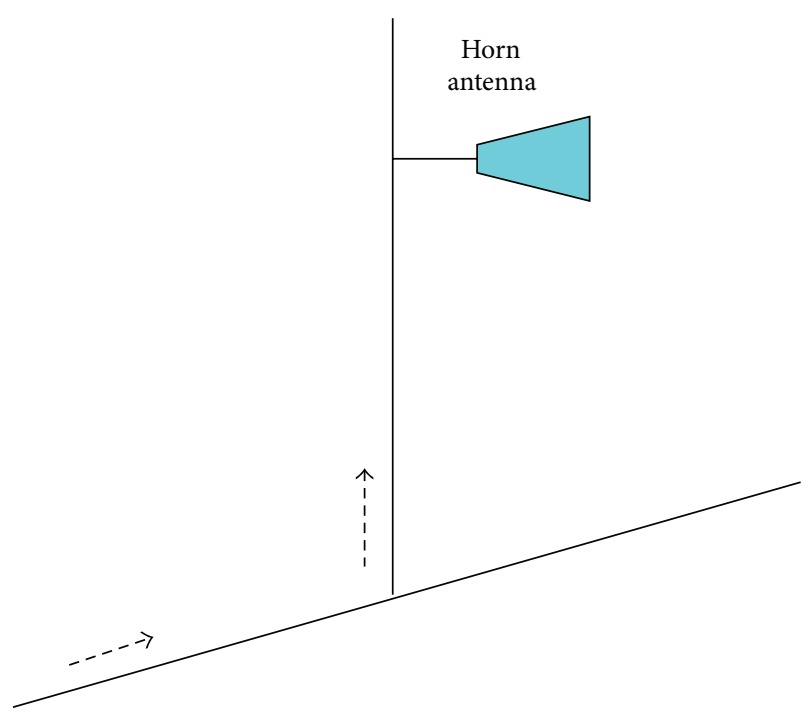

(b)

FIGURE 4: 3D EM imaging technical solutions: on the right, single antenna on a 2-axis translator; on the left, a fixed 2D antenna array.

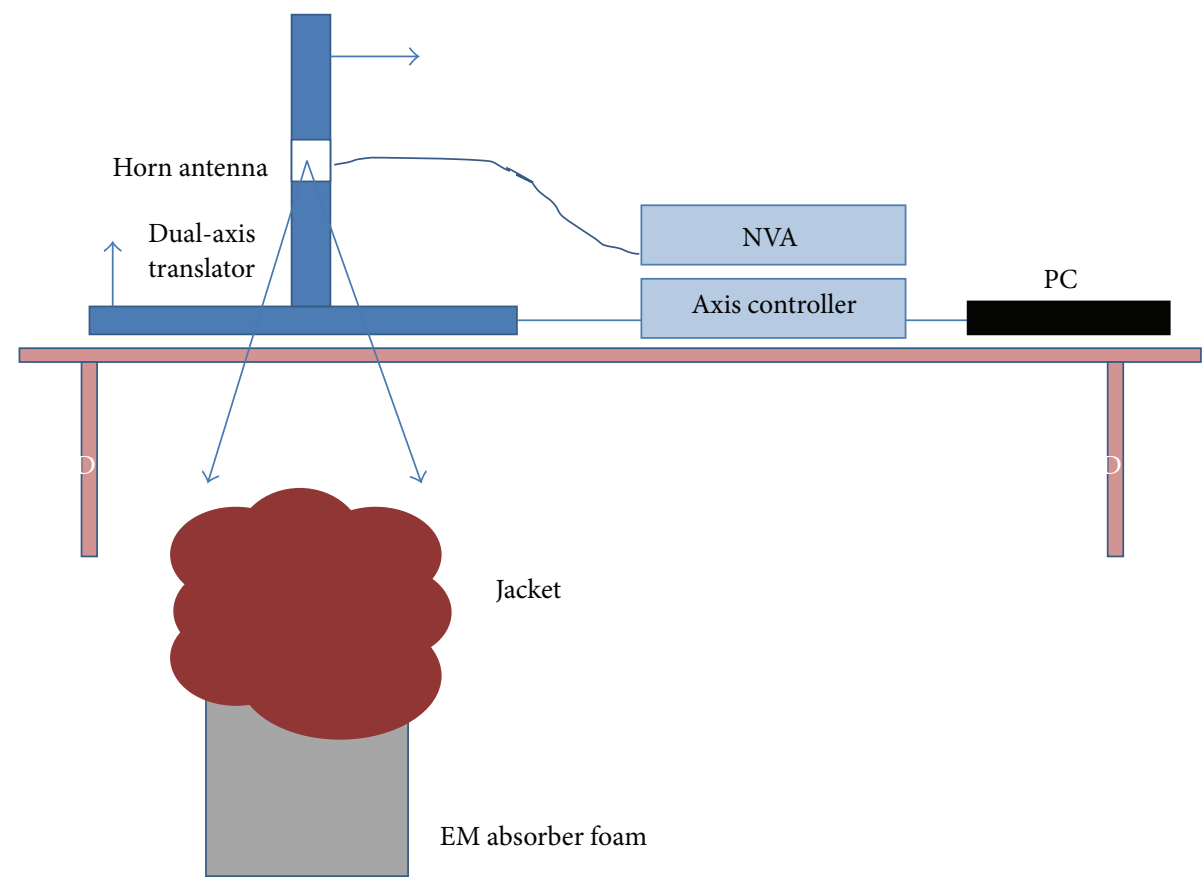

FIGURE 5: Schematic diagram of the imaging tool in operation.

The whole detection remains good. One can see that the metallic handle disappears a little but not the blade. The reason is the following. Due to the amount of water contained in the shirt, the shirt provides a large electromagnetic backscattering of large extent in the $(X, Y)$ plane. As the metal is in contact with the wet shirt, the processing that subtracts the mean value is partially confused. The blade that is not in contact with the shirt appears clearly on the image due to the $Z$-axis resolution capabilities of 3D imaging (see Figure 10).

\section{Randomly Sparse Synthetic Array Imaging}

In order to decrease the very long measurement time, one can imagine synthetizing a large sparse array [9]. The idea is to skip antenna measurements points along $X$ and $Y$ to go faster. A configuration has been found with a random distribution of elementary antennas. This configuration needs about $10 \%$ only of the full number of antenna points. The selection of the configuration is made by the following procedure: 
TABLE 3: List, description, and photographs of imaged objects.

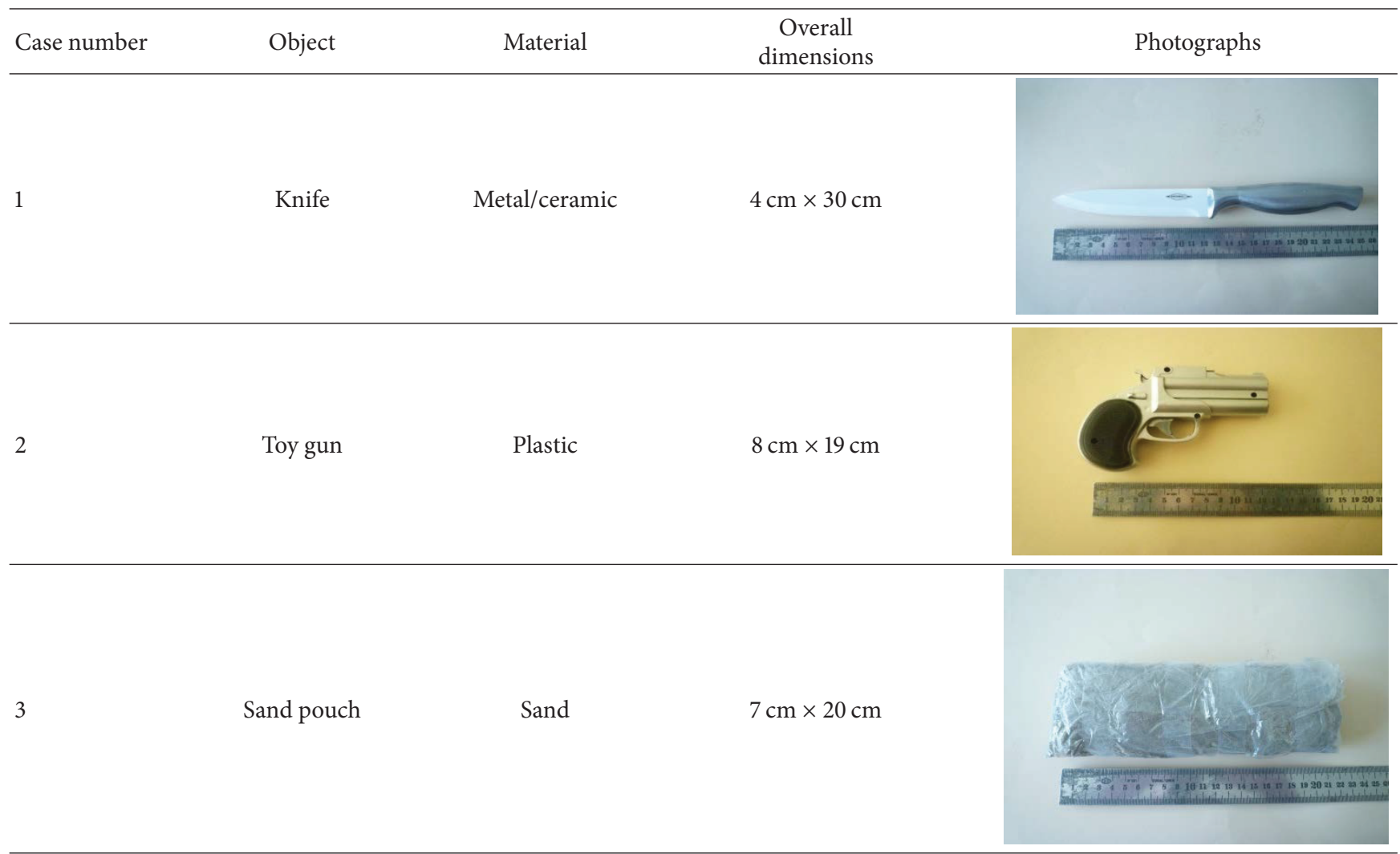

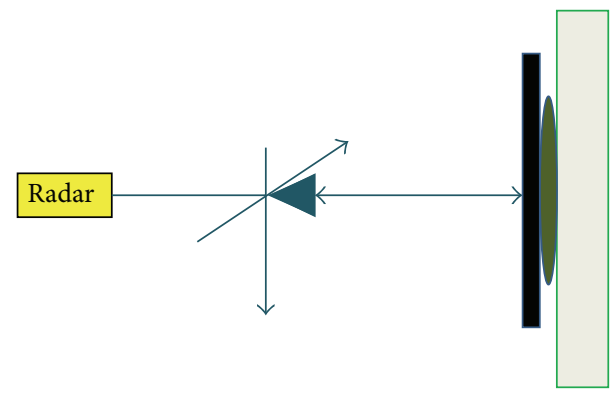

Figure 6: Configuration of the measurement (object is maintained between the coat and the absorber).

(i) Create a sparse matrix ("mask") of 0 and 1 of dimensions $51 * 51$ with $x \%$ sparsity.

(ii) Compute the response of a point-like target by simulation at a distance of $40 \mathrm{~cm}$ in near field.

(iii) Compute the response of the imaging processing by turning on and off the elementary radiators following the random rule given by the mask.

(iv) Adopt it according to a criterion of artifact level (e.g., $-15 \mathrm{~dB}$ of energy under the maximum peak).

With $x=10 \%$ sparsity, one can already find good representations. In this case, a mask is represented in Figure 11 and its image is given in Figure 12 (corresponding to the case of Figure 8).

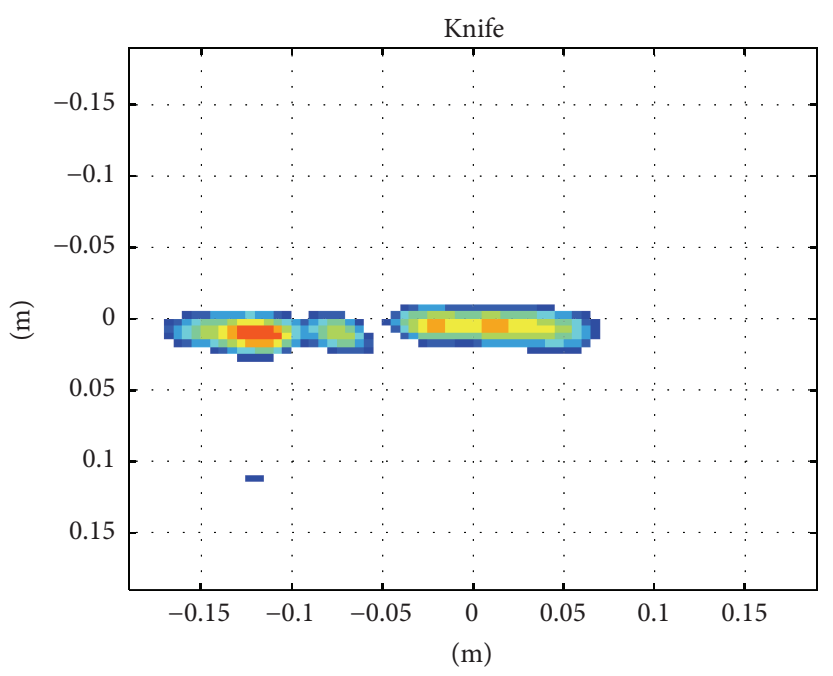

Figure 7: Ceramic/metal knife under coat (horizontal).

\section{Conclusion}

Short range UWB microwave imaging has been developed in $\mathrm{X}$-band and has proved its interest. It enables vision through clothes of objects of more than roughly $10 \mathrm{~cm}$ dimensions. Shapes can be estimated even though the image quality is not the same as optics or $\mathrm{THz}$ because of the wavelength. The interesting point is that we have found that the transmission 


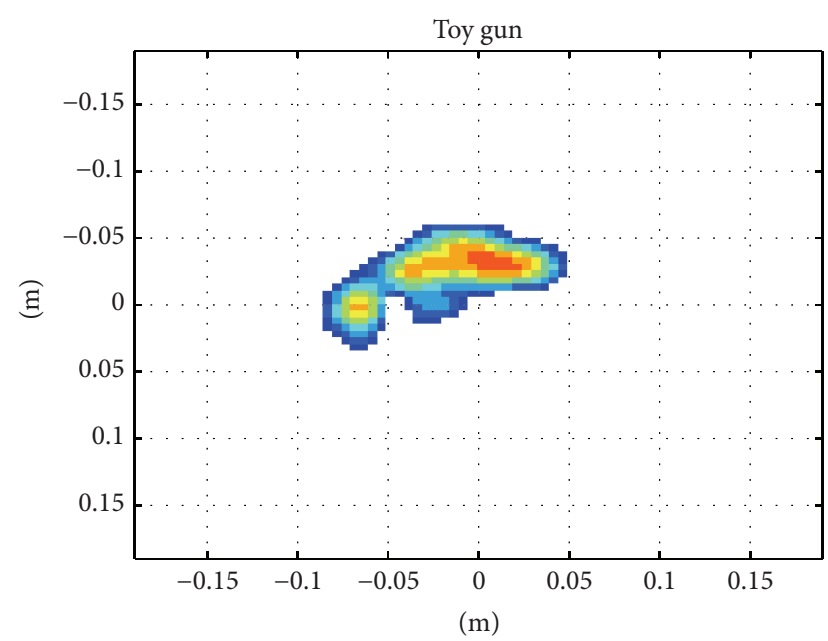

Figure 8: Plastic toy gun (horizontal).

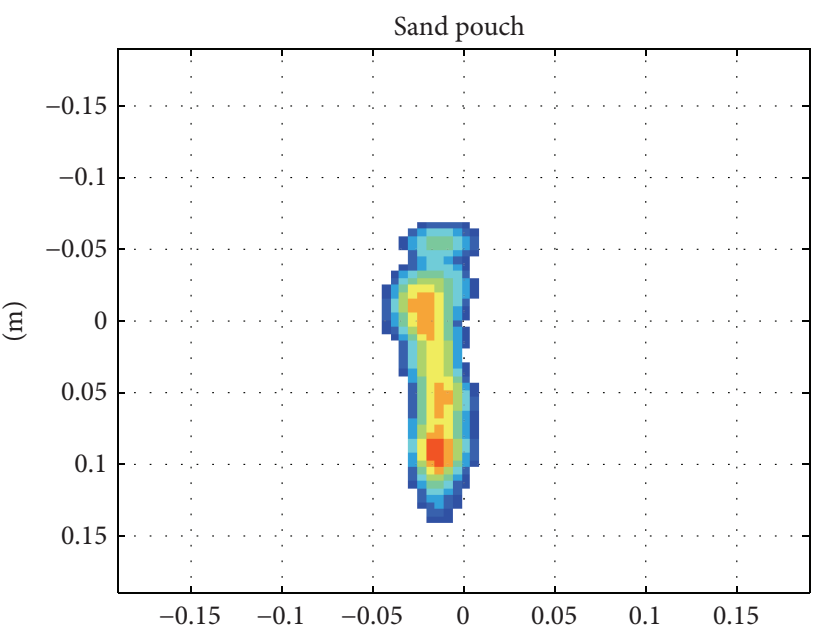

(m)

Figure 9: Sand pouch under coat (vertical).

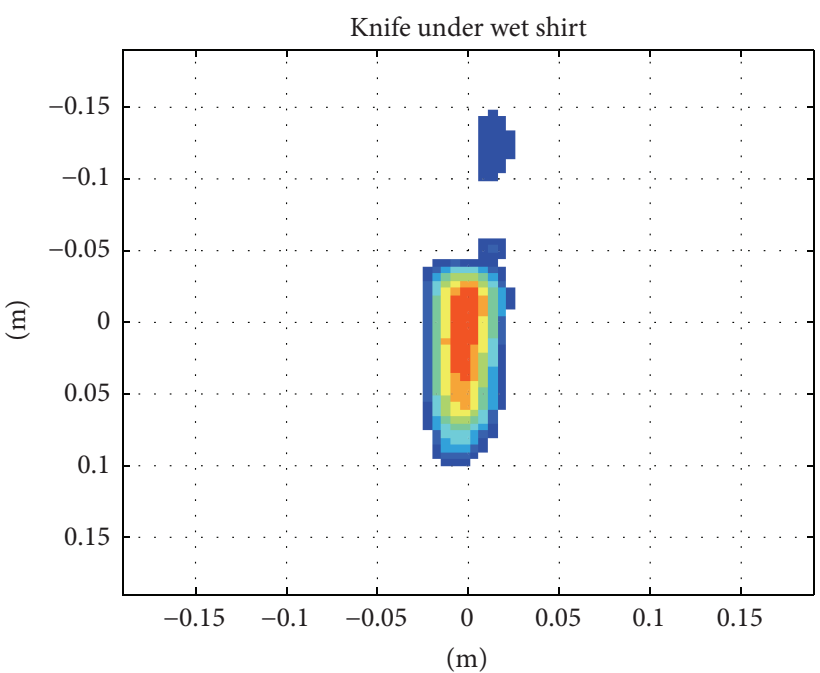

Figure 10: Knife under wet shirt (vertical).

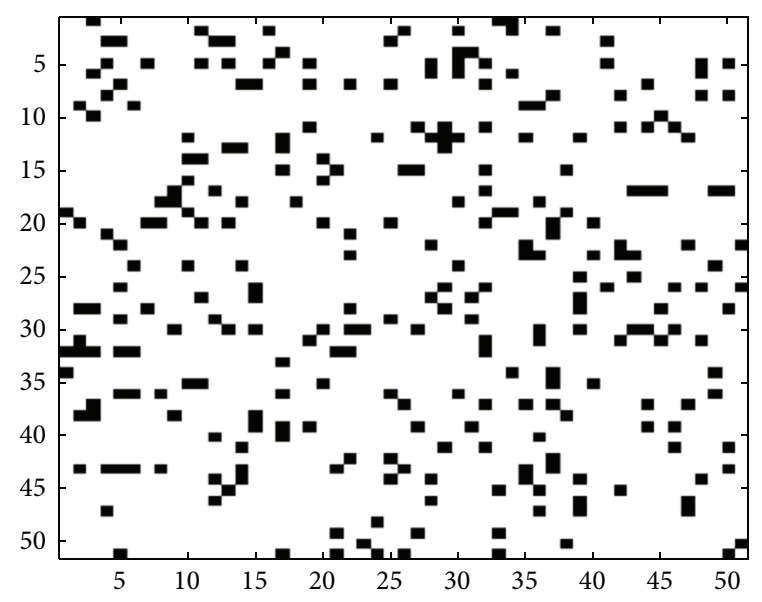

FIGURE 11: Sparse antenna array configuration: in black $(1=$ on $)$; in white $(0=$ off $)$.

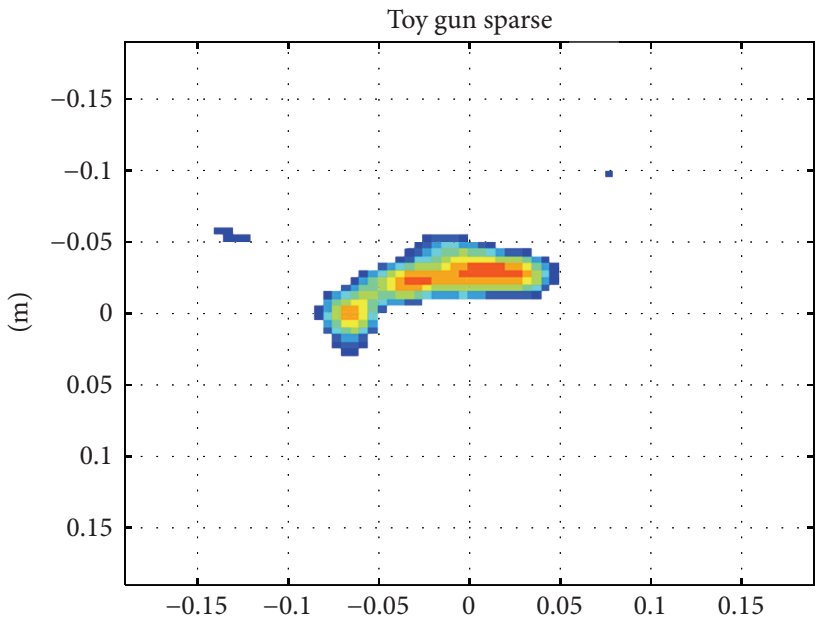

(m)

FIGURE 12: Toy gun, with very sparse configuration.

through clothes, even slightly wet, does not disturb the images and so inexpensive microwave systems could be envisaged. The main drawback comes from the measurement time. At this time, it is not possible to make measurements with a real person. Signal processing computation time is no more a problem with 3D-SAR clever formulations. In order to improve the measurement time, a research axis has been opened. Sparse array configurations already give good results (even with some unwanted side-lobes) and the researches are ongoing in the field of electromagnetic imaging algorithms. Three new ideas are emerging:

(i) The first one is technical: it consists in using an arc with antennas (equipped with digital switches) and to sweep the human body along a circle as a scanner. For the purpose, a line of antennas (like open waveguides) can been used as well as miniaturized FMCW radar heads. 
(ii) The second one is the MIMO-SAR or sparse configuration-both on transmission and on receptionfor the antenna array, which may bring about another drastic decreasing of the number of measurements at the price of a reduced signal-to-noise ratio.

(iii) Last, microwave imaging at $\mathrm{mm}$ wave will also be performed in order to fuse information between $\mathrm{X}$ band and $\mathrm{mm}$ wave $(60 \mathrm{GHz})$.

\section{Conflict of Interests}

The authors declare that there is no conflict of interests regarding the publication of this paper.

\section{Acknowledgment}

The authors are very grateful to the European Defense Agency for having partially funded the ONERA Lab for explosive detection studies in the framework of the E-STAR project.

\section{References}

[1] D. M. Sheen, D. L. McMakin, and T. E. Hall, "Three-dimensional millimeter-wave imaging for concealed weapon detection," IEEE Transactions on Microwave Theory and Techniques, vol. 49, no. 9, pp. 1581-1592, 2001.

[2] R. Appleby and H. B. Wallace, "Standoff detection of weapons and contraband in the $100 \mathrm{GHz}$ to $1 \mathrm{THz}$ region," IEEE Transactions on Antennas and Propagation, vol. 55, no. 11, pp. 29442956, 2007.

[3] G. Gennarelli, I. Catapano, F. Soldovieri, and R. Persico, "On the achievable imaging performance in full 3-D linear inverse scattering," IEEE Transactions on Antennas and Propagation, vol. 63, no. 3, pp. 1150-1155, 2015.

[4] R. Solimene, A. Brancaccio, R. Di Napoli, and R. Pierri, "3D sliced tomographic inverse scattering experimental results," Progress in Electromagnetics Research, vol. 105, pp. 1-13, 2010.

[5] A. H. Golnabi, P. M. Meaney, N. R. Epstein, and K. D. Paulsen, "Microwave imaging for breast cancer detection: advances in three-dimensional image reconstruction," in Proceedings of the 33rd Annual International Conference of the IEEE Engineering in Medicine and Biology Society, pp. 5730-5733, Boston, Mass, USA, August-September 2011.

[6] S. E. Assad, I. Lakkis, and J. Saillard, "Holographic SAR image formation by coherent summation of impulse response derivatives," IEEE Transactions on Antennas and Propagation, vol. 41, no. 5, pp. 620-624, 1993.

[7] M. Soumekh, Synthetic Aperture Radar Signal Processing, John Wiley \& Sons, 1999.

[8] J. Fortuny and A. J. Sieber, "Fast algorithm for a near-field synthetic aperture radar processor," IEEE Transactions on Antennas and Propagation, vol. 42, no. 10, pp. 1458-1460, 1994.

[9] Y. Qi, Y. Wang, X. Peng, W. Tan, and W. Hong, "Application of optimized sparse antenna array in near range 3D microwave imaging," in Proceedings of the 17th International Symposium on Antennas and Propagation (ISAP'12), pp. 1397-1400, Nagoya, Japan, November 2012. 

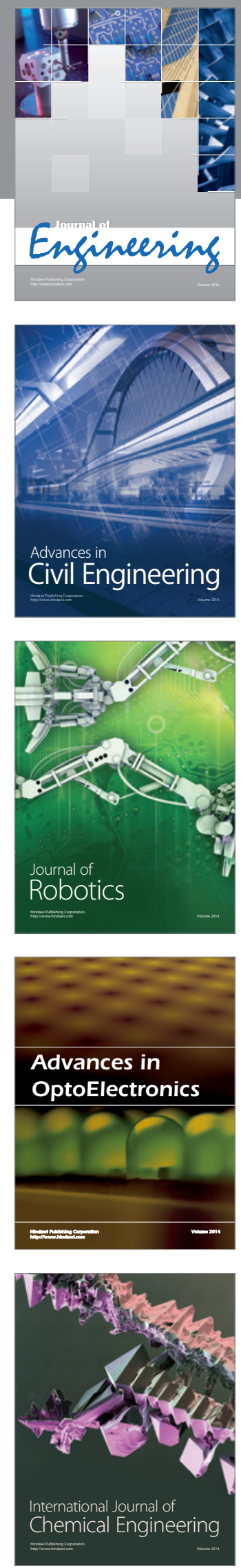

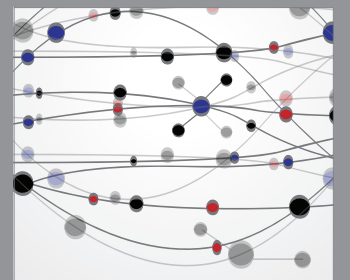

The Scientific World Journal
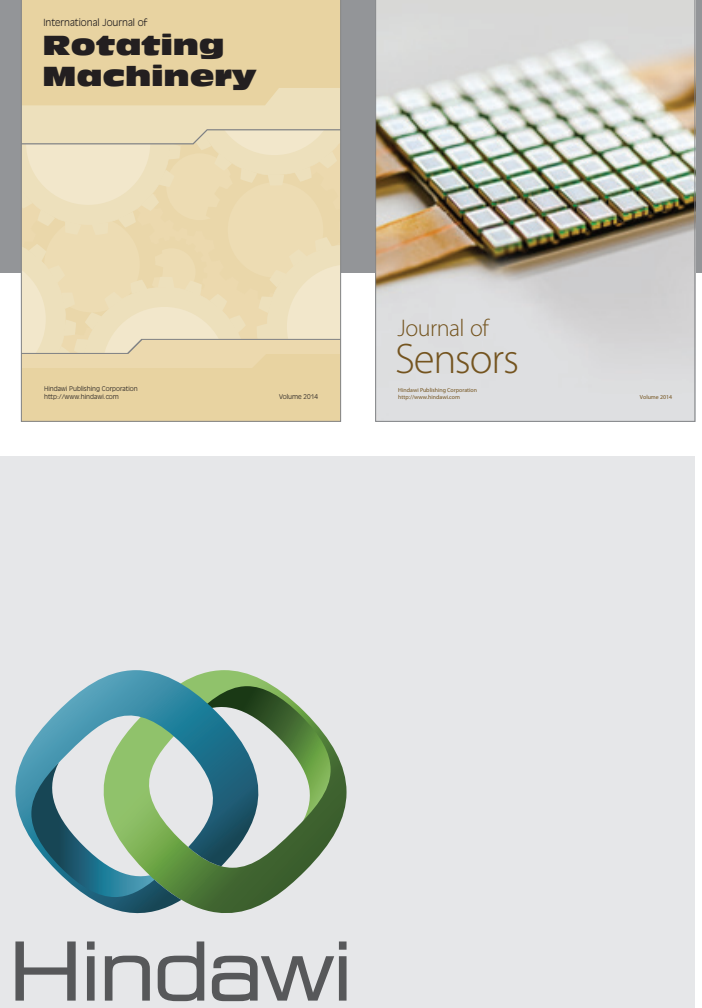

Submit your manuscripts at http://www.hindawi.com
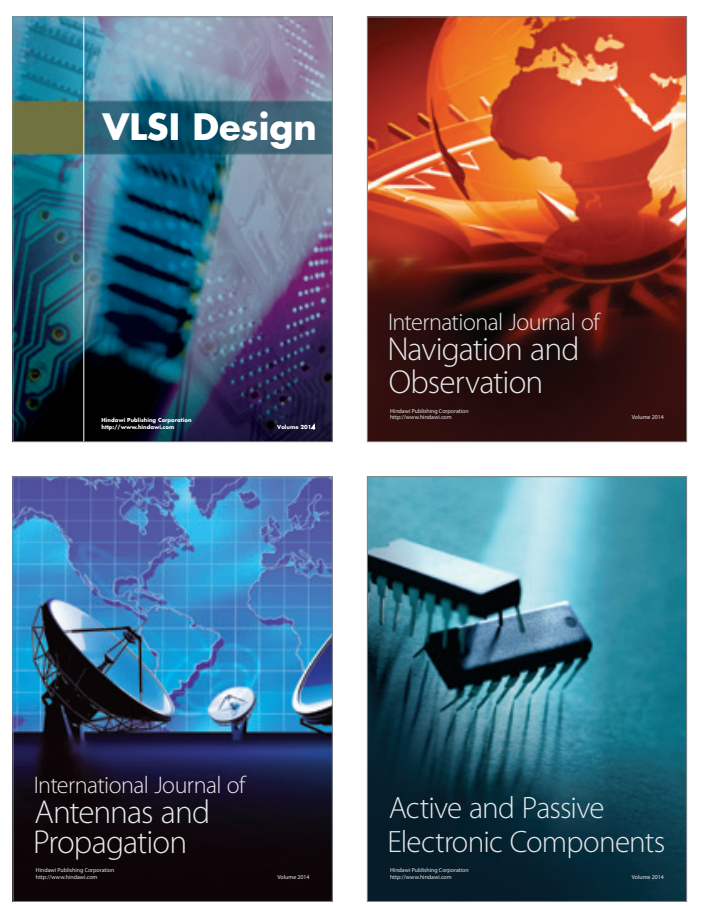
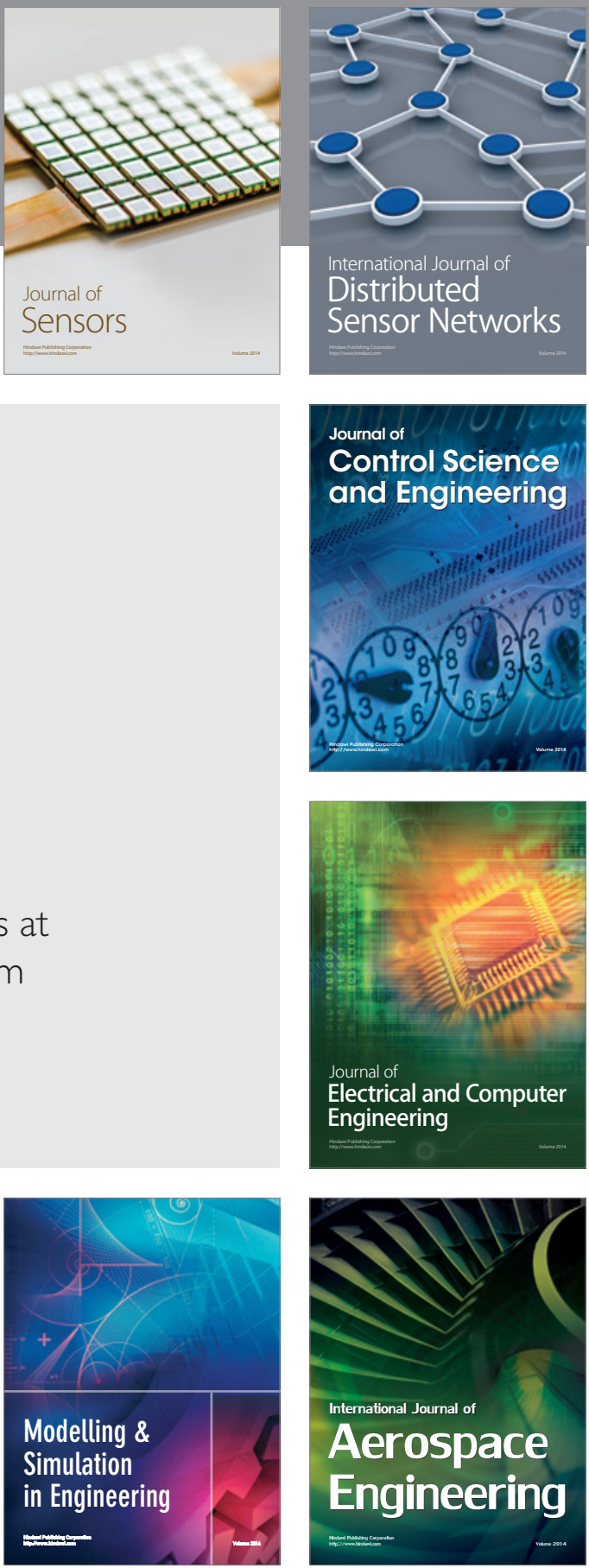

Journal of

Control Science

and Engineering
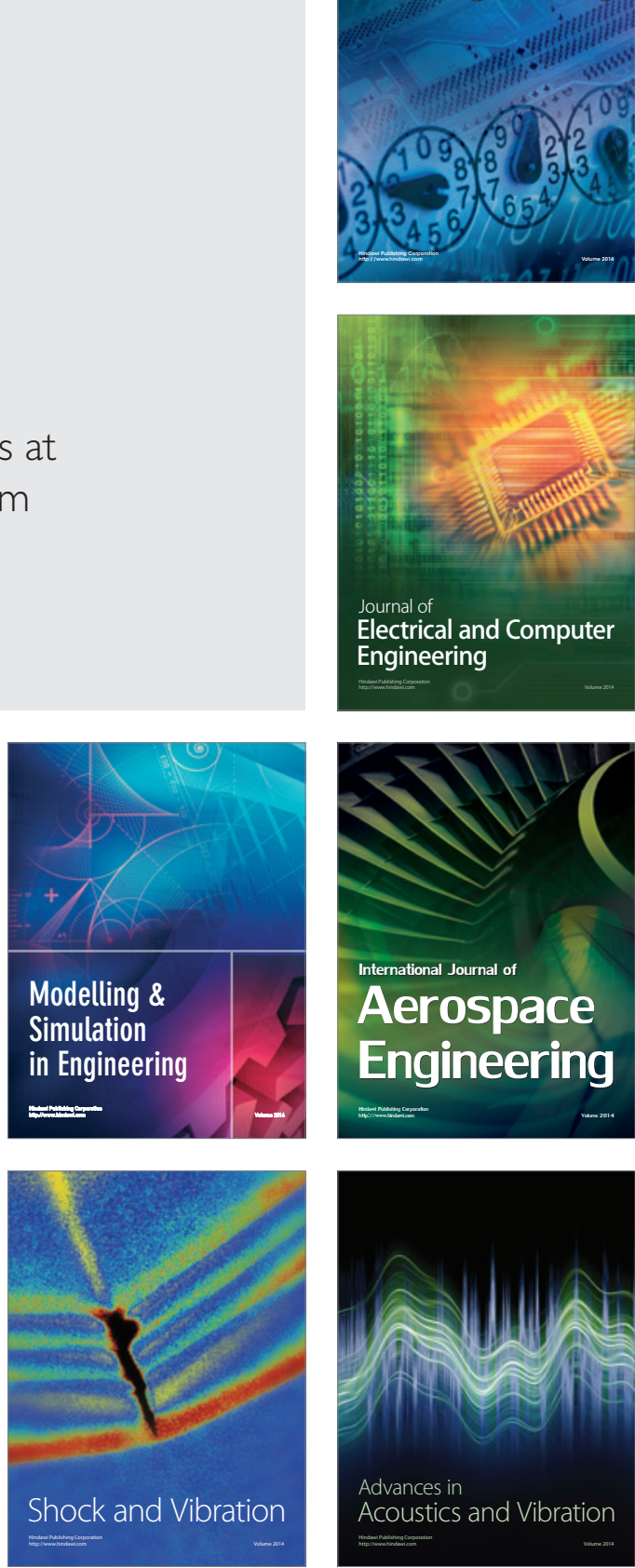